\title{
Conversion Reaction of Polyoxometalates from Anderson Structure to Keggin Structure
}

\author{
Teruyoshi YAMAKAWA, ${ }^{\mathrm{a}, *}$ Kazuo EDA,,${ }^{\mathrm{a},}$ Toshiyuki OSAKAI, ${ }^{\mathrm{a}}$ Takahito NAKAJIMA ${ }^{\mathrm{b}}$
}

\author{
${ }^{a}$ Graduate School of Science, Kobe University, 1-1, Rokkodai-cho, Nada-ku, Kobe, 657-8501, Japan \\ ${ }^{b}$ RIKEN Center for Computational Science, 7-1-26 Minatojima-minamimachi, Chuo-ku, Kobe, Hyogo, 650-0047, Japan \\ *181s225s@stu.kobe-u.ac.jp,eda@kobe-u.ac.jp
}

(Received: December 11, 2019; Accepted for publication: January 16, 2020; Online publication: April 9, 2020)

Aiming at developing synthetic methods of new Keggin-type polyoxometalates (POMs) that can mediate protonconjugated multi-electron transfer reactions useful for efficient regeneration of fuels, we have investigated the reaction routes from Anderson-type POMs to Keggin-type POMs. The POM systems with various kinds of heteroatoms as their central cations were calculated using the Nudged Elastic Band (NEB) method as well as the first-principles electronic structure method. The effects of the heteroatoms on the reaction routes have been discussed.

Keywords: Polyoxometalates, Polyoxotungstates, NEB

\section{Introduction}

Keggin-type polyoxometalates (POMs) have a spherical closed-metal-oxide shell structure and their heteroatoms $\left(X{ }^{\top} \mathrm{s}\right)$ are located inside of the shell (Figure 1). So their properties such as redox potentials and basicity can be controlled with the kind of heteroatom without influencing their ionic radius and surface structure. They are stable and can receive many electrons and protons without destroying their structure. Thus they become good reservoirs of electrons and protons and are expected as candidates of catalysts that can promote the proton-coupled multi-electron transfer reactions such as $\mathrm{CO}_{2}+4 \mathrm{H}^{+}+4 \mathrm{e}^{-} \rightarrow \mathrm{C}+2 \mathrm{H}_{2} \mathrm{O}$ and $\mathrm{C}+4 \mathrm{H}^{+}+4 \mathrm{e}^{-} \rightarrow \mathrm{CH}_{4}$ (fuels' regeneration reactions).

In order to realize the catalysts, we have studied synthetic routes for new Keggin-type POMs that can mediate the multielectron transfer reactions. Previously we have revealed that Keggin-type POMs are formed from Anderson-type POMs via a structural conversion between the two kinds (Andersonand Keggin-type) of six-membered rings by intramolecular hydrolysis and condensation processes [1]. In this study we have investigated the reaction routes from Andersontype POMs to Keggin-type POMs using the Nudged Elastic
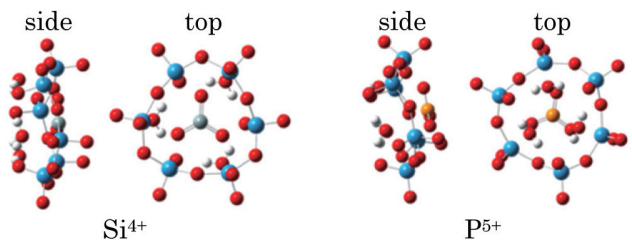

$\mathrm{P} 5+$

Figure 1. Changes in the $\mathrm{I}_{\mathrm{f}}$ structure with the ionic charge of heteroatoms; side: side view, top: top view.

Band (NEB) method as well as the first-principles electronic structure method in order to obtain clues for preparation of new Keggin-type POMs.

\section{Computational methods}

The conversion routes of Anderson-type POMs with various kinds of heteroatoms (Anderson-type six- membered W-O rings $\mathrm{I}_{\mathrm{i}} \mathrm{s},\left[X(\mathrm{OH})_{6} \mathrm{~W}_{6} \mathrm{O}_{18}\right]^{z} ; X=\mathrm{Ni}, \mathrm{Zn}, \mathrm{B}, \mathrm{Al}, \mathrm{Co}, \mathrm{Ga}$, $\mathrm{Si}, \mathrm{Ge}, \mathrm{P}, \mathrm{As}, \mathrm{S})$ to the Keggin-type six-membered rings $\mathrm{I}_{\mathrm{f}} \mathrm{S}$ $\left(\left[X \mathrm{O}_{3} \mathrm{~W}_{6} \mathrm{O}_{18}\left(\mathrm{H}_{2} \mathrm{O}\right)_{3}\right]^{z}\right)$ were dealt with. The initial structure of $\mathrm{I}_{\mathrm{f}}$ was obtained by removing two $\mathrm{W}_{3}$ units from the whole structure of Keggin-type POM (Figure S1). For the first-principles electronic structure and NEB methods the comprehensive software package NTChem [2] was used. The 
geometry optimizations were performed without symmetry constraints ( $C_{1}$ symmetry) using a program library DL-find [3]. All calculations were carried out using the density functional theory $\omega \mathrm{B} 97 \mathrm{XD}$ and the basis set Def2-SVP. Effective core potentials were used for $\mathrm{W}$ atoms only. The computations were performed using Research Center for Computational Science, Okazaki, Japan and an OTB Xeon-HW-08 workstation equipped with two 12 core CPUs.

\section{Results and discussion}

The structural optimization calculations of $\mathrm{I}_{\mathrm{i}}$ 's (Andersontype POMs) showed less dependence of their resulting structure on the cationic charge $n$ of the heteroatoms, while those of If's had apparent dependence. The resulting If's having heteroatoms with $n$ ranging from 2 to 4 exhibit trigonal riceball (trb) shapes intrinsic for Keggin-type six-membered rings, but those having heteroatoms with $n$ of 5 and 6 show round rice-ball (rrb) shapes (Table S1). Comparing between total energies of corresponding $\mathrm{I}^{\prime}$ 's and $\mathrm{I}_{\mathrm{f}} \mathrm{s}$, the sign of $\Delta E_{\text {total }}$ ( $=E_{\text {total,If }}-E_{\text {total,Ii }}$ changes from positive to negative when the ring type of $I_{f}$ turn from the trb shape to the rrb one (Table $\mathrm{S} 1)$. In the $\mathrm{I}_{\mathrm{f}}$ with the rrb shape desertion of the heteroatom unit $\left[\mathrm{XO}_{3}\right]^{n-6}$ from the six-membered ring occurs, as shown in Figure 1. Such desertion is probably observed when the energy gain due to the electrostatic interaction between the heteroatom unit and positively polarized inner surface of the (W-O) ring cannot exceed the energy required for ring deformation from rrb to trb, induced by insertion of the unit. Since we have found out that the Keggin-type POMs having heteroatoms with smaller cationic charge and larger ionic radius may enable proton-coupled multi-electron transfer [4], these findings may become clues for preparation of the target Keggin-type POMs.

Reaction path searches using these $\mathrm{I}_{\mathrm{i}}$ 's and $\mathrm{I}_{\mathrm{f}}$ 's are hard to be converged and still in progress. Figure 2 shows one of the calculation results (reaction path search for $X=\mathrm{Si}$ in gas-phase with 5 images, using a loose ThrGMax convergence criterion of 0.0025 in atomic units). The transition state was calculated by the Climbing Image method after the normal NEB search. The results show two saddle points: the initial with small uphill and high activation barrier, and the subsequent with larger uphill and much smaller barrier. Comparing the structural changes to the two points, the former involves breaking the six-membered ring, while the latter does not and is related to

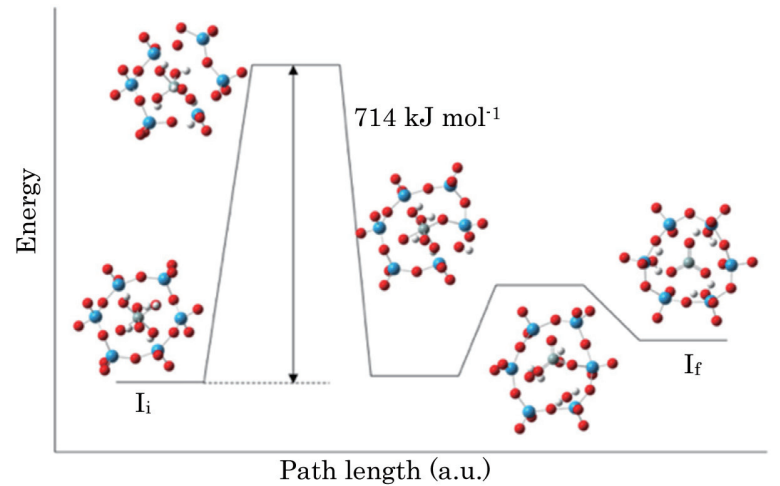

Figure 2. Exploration results of the conversion path between Anderson-type and Keggin-type six-membered rings $\mathrm{I}_{\mathrm{i}}$ and $\mathrm{I}_{\mathrm{f}}$, for $X=\mathrm{Si}$.

dehydration process, which requires much smaller energy. The initial activation barrier is high, $714 \mathrm{~kJ} \mathrm{~mol}^{-1}$ and indicates the requirement of reducing this high activation energy for preparation of target POMs.

\section{Conclusion}

We have investigated the structural conversion from Anderson-type rings to Keggin-type rings using the firstprinciples electronic structure and the NEB methods. The apparent dependences of $\Delta E_{\text {total }}$ (between the two rings) and of the latter ring structure on the heteroatom's cationic charge were observed. The NEB search of the conversion path showed two saddle points and a very high activation energy.

\section{Supplementary Materials}

Supporting_information.pdf(Figure S1 and Table S1)

\section{Acknowledgement}

This work was supported by JSPS KAKENHI Grant Number JP18K05274.

\section{References}

[1] S. Himeno, S. Murata, K. Eda, Dalton Trans., 6114 (2009). DOI:10.1039/b902794g

[2] T. Nakajima, M. Katouda, M. Kamiya, Y. Nakatsuka, Int. J. Quantum Chem., 115, 349 (2015). DOI:10.1002/ qua. 24860

[3] J. Kästner, J. M. Carr, T. W. Keal, W. Thiel, A. Wander, P. Sherwood, J. Phys. Chem. A, 113, 11856 (2009). DOI:10.1021/jp9028968, PMID:19639948

[4] K. Eda, T. Osakai, Inorg. Chem., 54, 2793 (2015). DOI:10.1021/ic502970q, PMID:25741616 